\title{
Expression and localization of the vascular endothelial growth factor and changes of microvessel density during hair follicle development of Liaoning cashmere goats
}

\author{
Q.L. Zhang ${ }^{1}$, J.P Li ${ }^{2}$, Y.M. Li ${ }^{1}$, Q. Chang ${ }^{2}$, Y. Chen ${ }^{2}$, H.Z. Jiang ${ }^{2}$, \\ Z.H. Zhao ${ }^{1}$ and D. Guo ${ }^{3}$ \\ ${ }^{1}$ College of Animal Science and Veterinary Medicine, Jilin University, \\ Changchun, Jilin, China \\ ${ }^{2}$ College of Animal Science and Technology, Jilin Agricultural University, \\ Changchun, Jilin, China \\ ${ }^{3}$ Liaoning Cashmere Goat Breeding Center, Liaoyang, China \\ Corresponding authors: H.Z. Jiang / Z.H. Zhao \\ E-mail: jianghz6806@126.com / zhzhao@jlu.edu.cm
}

Genet. Mol. Res. 12 (4): 6424-6432 (2013)

Received April 9, 2013

Accepted August 15, 2013

Published December 10, 2013

DOI http://dx.doi.org/10.4238/2013.December.10.3

\begin{abstract}
Vascular endothelial growth factors (VEGFs) play important roles in neovascularization, tissue development, and angiogenesis. In this study, changes in VEGF expression patterns and microvessel density (MVD), and their correlations, were investigated during hair follicle development in epidermal appendages of Liaoning cashmere goats. Polyclonal antibodies to VEGF and microvessels were used for monthly immunohistochemical examinations of normal skin specimens from adult female goats for one year. VEGF was expressed in the hair bulb of primary and secondary hair follicles, the outer and inner root sheaths, sebaceous glands (ductal and secretory portions), eccrine sweat glands (ductal and secretory portions), and the epidermis. Abundant expression of VEGF was observed in the follicular basement membrane
\end{abstract}


zone surrounding the bulb matrix and in ductal and secretory portions of eccrine sweat glands. The change in VEGFs in primary hair follicles showed a bimodal pattern, with the first peak observed from March to May, and the second in August. Maximal expression in secondary hair follicles occurred in May and August. Therefore, VEGF expression in primary and secondary hair follicles is synchronized throughout the year, and is correlated to hair development. In the later telogen and anagen phases, VEGF expression was higher in the secondary, compared to the primary, hair follicle. Changes in MVD also showed a bimodal pattern with peaks in May and August. VEGF expression and MVD showed moderate and strongly positive correlation in the primary and secondary hair follicles, respectively. Therefore, MVD and VEGF are closely related to the processes involved in hair cycle regulation.

Key words: Adult Liaoling cashmere goats; VEGF; MVD; Hair follicle cycle

\section{INTRODUCTION}

The vascular endothelial growth factor (VEGF), also known as the vascular permeability factor (Viac et al., 1997), is a specific mitogen for vascular endothelial cells (VECs) (Roy et al., 2006). For normal cell growth and differentiation, an adequate supply of blood appears to be of fundamental importance in the active processes of hair growth. The dermal papilla of the hair follicle, containing follicular stem cells, presents well-developed vascularization by providing optimal growth conditions (Kozlowska et al., 1998a). Previous studies have shown that hair vasculature undergoes hair cycle-dependent changes: anagen human hair follicles are highly vascularized while most capillaries collapse and disappear during catagen (Borodach and Montagna, 1956; Ellis and Moretti, 1959; Chase, 1965). The first changes in the early catagen phase occur around the small vessels of the dermal papilla. Dermal papillae are small, mesenchymally derived zones at the bases of hair follicles, and play important roles in hair morphogenesis, embryonic development, and control of the hair growth cycle in postnatal mammals (Couchman, 1986; Reynolds and Jahoda, 1996). However, the hypothesis that the hair follicle enters catagen due to decreased vascularization has not yet been confirmed (Parakkal, 1990). Indeed, the responsiveness of skin to angiogenic stimuli depends on the stage of the hair cycle, with maximal responsiveness observed during the anagen VI stage in mouse skin (Majewski et al., 1985).

Hair follicle vascularization appears to be closely related to the processes involved in hair cycle regulation, which involve growth factors, cytokines, and other bioactive molecules (Kozlowska et al., 1998b). In particular, VEGF plays important roles in promoting angiogenesis by stimulating capillary proliferation, migration, and permeability, as well as tissue development, by regulating growth and differentiation (Ferrara et al., 2003; Tammela et al., 2005). VEGF expression has been observed in normal epidermis (Ballaun et al., 1995; Weninger et al., 1996), skin tumors (Weninger et al., 1996; Viac et al., 1997), hair follicles (Man et al., 2009), and sebaceous glands (Lachgar et al., 1996a,b; Kozlowska et al., 1998b). The latter two, together with eccrine sweat glands, are termed the 'epidermal appendages', and are lined with epithelial cells (Man et al., 2009). Therefore, VEGF may be responsible for maintaining 
proper vasculature around the hair follicle during the anagen growth phase.

To date, no study has yet focused on the distribution, dynamics, or relationship between VEGF and microvessel density (MVD) during the hair cycle of Liaoning cashmere goats, although the expression of VEGF in hair follicles and sebaceous glands have been previously documented. This study aimed to analyze the expression and distribution of VEGF, the changes of MVD, and their dynamics during the hair cycle of Liaoning cashmere goats, and to investigate whether there is variation across different periods of hair follicle development.

\section{MATERIAL AND METHODS}

\section{Specimens}

This study was approved by the Liaoning Provincial Cashmere Goat Breeding Center. Five 1.5-year-old Liaoning cashmere goats were selected randomly in November 2009, which were depastured alongside a pristine goat herd without any additional treatment. Approximately $3 \mathrm{~cm}$ healthy skin specimens were obtained from the right side of the scapula by biopsy punch $(1 \mathrm{~cm}$ diameter) once a month and handled aseptically.

\section{Immunohistochemistry}

The specimens were fixed in $4 \%$ paraformaldehyde, processed through graded concentrations of ethanol, and the uppermost epidermal surfaces were embedded (YD6D) in paraffin wax. Four micrometer sections of embedded skin were serially cut in the transverse plane on a rotary microtome (Leica RM 2235), deparaffinized, and rehydrated. Antigen retrieval was performed by incubating the sections in $0.01 \mathrm{M}$ citrate buffer $(\mathrm{pH} 6.0)$ at $90^{\circ} \mathrm{C}$ for $10 \mathrm{~min}$, and by cooling naturally at room temperature for $20 \mathrm{~min}$. The sections were incubated with an optimal dilution each primary antibody (Table 1). After rinsing, biotinylated antibody immunoglobulin $\mathrm{G}$ and a complex of streptavidin peroxidase were added in sequence, according to manufacturer instructions. Negative controls were carried out by replacing the primary antibody with phosphate buffered solution as the anti-VEGF (bs-0279R) and antiCD34 antibody (bs-0646R). The visualization system included 3,3'-diaminobenzadine solution, counterstained with hematoxylin.

Table 1. Concentration, time, and condition of incubation for different primary antibodies.
\begin{tabular}{lccc}
\hline Primary antibody & Antibody concentration & Time & Temperature \\
\hline VEGF & $10 \mu \mathrm{g} / \mathrm{mL}$ & $10 \mathrm{hr}$ & $4^{\circ} \mathrm{C}$ \\
CD34 & $5 \mu \mathrm{g} / \mathrm{mL}$ & $10 \mathrm{hr}$ & $4^{\circ} \mathrm{C}$ \\
\hline
\end{tabular}

\section{Statistical analyses}

The average optical density (OD) of VEGF was analyzed using the IPP 6.0 software. Areas of interest (AOI) were selected to investigate the expression of antigens in the primary and secondary hair follicles by measuring the AOI, revising the OD, selecting the color of the positive area, and setting the analysis conditions to calculate the average OD of the AOI. 
The data obtained represents the expression intensity of the antigen at a particular period. The MVD was measured by marking VECs with a CD34 antibody.

The means and standard deviations of relevant data were statistically analyzed using a completely randomized design with the SPSS Statistics 18 (SPSS 18.0) software. Analysis of variance of single factors and multiple comparisons were performed using the Duncan method, and correlations were determined using the Pearson method.

\section{RESULTS}

\section{Immunohistochemical expression of VEGF across different phases}

Results of analyses of the longitudinal section of VEGFs of adult female Liaoning cashmere goats across different months, the average OD of VEGFs in the primary and secondary hair follicles, and MVD (Table 2) revealed that VEGFs were expressed in the hair follicle (including the inner and outer root sheaths [IRS and ORS, respectively] and the dermal papillae epidermal matrix), sebaceous glands (ductal and secretory portions), and eccrine sweat glands (ductal and secretory portions) (Figure 1). The average OD of VEGFs varied in the primary hair follicle and the curve showed a bimodal pattern of change. The first peak occurred in March, April, and May, and the second was in August (0.046 \pm 0.001$)$, with no significant difference between these two periods $(\mathrm{P} \geq 0.05)$. The first peak decreased substantially in June and July $(\mathrm{P} \leq 0.05)$, and then increased significantly in August $(\mathrm{P} \leq 0.05)$, while the second peak was maintained for only one month and then decreased significantly $(\mathrm{P} \leq 0.05)$. The average ODs of VEGFs in the secondary hair follicle were similar with those of the primary hair follicle, and followed a bimodal pattern of change (Figure 2). The average OD of VEGFs increased gradually from January to April, but the change was not significant $(\mathrm{P} \geq 0.05)$ until it increased abruptly in May $(\mathrm{P} \leq 0.05)$ and reached the first peak $(0.052 \pm$ $0.008)$. VEGFs then decreased significantly in June $(P \leq 0.05)$, and then increased significantly in August $(\mathrm{P} \leq 0.05)$, reaching the second peak $(0.049 \pm 0.011)$, which was maintained until October $(\mathrm{P} \geq 0.05)$. However, in the later telogen and pre-anagen phases, the expression of VEGF in the secondary hair follicle was higher than that in the primary hair follicle

$\begin{aligned} & \text { Table 2. Average optical of VEGF on hair follicle and MVD in dermis of adult female Liaoning cashmere goats } \\
& \text { in different months (means } \pm \text { SD). }\end{aligned}$
\begin{tabular}{lccc}
\hline Month & $\begin{array}{c}\text { Average optical of VEGF } \\
\text { Primary hair follicle }\end{array}$ & Secondary hair follicle & Microvessel density (MVD) \\
\hline 1 & $0.013 \pm 0.011^{\mathrm{ab}}$ & $0.011 \pm 0.006^{\mathrm{a}}$ & $3.43 \pm 0.75^{\mathrm{a}}$ \\
2 & $0.023 \pm 0.005^{\mathrm{b}}$ & $0.017 \pm 0.002^{\mathrm{a}}$ & $4.05 \pm 0.50^{\mathrm{ab}}$ \\
3 & $0.042 \pm 0.006^{\mathrm{c}}$ & $0.025 \pm 0.007^{\mathrm{abc}}$ & $6.01 \pm 0.80^{\mathrm{d}}$ \\
4 & $0.044 \pm 0.005^{\mathrm{c}}$ & $0.033 \pm 0.008^{\mathrm{abc}}$ & $4.84 \pm 0.66^{\mathrm{bc}}$ \\
5 & $0.043 \pm 0.014^{\mathrm{c}}$ & $0.052 \pm 0.008^{\mathrm{c}}$ & $7.57 \pm 0.73^{\mathrm{e}}$ \\
6 & $0.024 \pm 0.011^{\mathrm{b}}$ & $0.023 \pm 0.013^{\mathrm{ab}}$ & $4.62 \pm 0.59^{\mathrm{abc}}$ \\
7 & $0.015 \pm 0.003^{\mathrm{a}}$ & $0.021 \pm 0.005^{\mathrm{a}}$ & $7.43 \pm 0.33^{\mathrm{bc}}$ \\
8 & $0.046 \pm 0.001^{\mathrm{c}}$ & $0.049 \pm 0.011^{\mathrm{bc}}$ & $5.38 \pm 0.54^{\mathrm{cd}}$ \\
9 & $0.029 \pm 0.007^{\mathrm{b}}$ & $0.034 \pm 0.004^{\mathrm{bc}}$ & $5.86 \pm 0.63^{\mathrm{d}}$ \\
10 & $0.028 \pm 0.001^{\mathrm{b}}$ & $0.039 \pm 0.007^{\mathrm{bc}}$ & $4.86 \pm 0.80^{\mathrm{bcd}}$ \\
12 & $0.031 \pm 0.001^{\mathrm{b}}$ & $0.026 \pm 0.004^{\mathrm{a}}$ & $3.85 \pm 0.26^{\mathrm{ab}}$ \\
\hline
\end{tabular}

The different letter showed that the difference was significant in the same column $(\mathrm{P}<0.05)$, and the equal letter showed that the difference was not significant in the same column $(\mathrm{P}>0.05)$. 


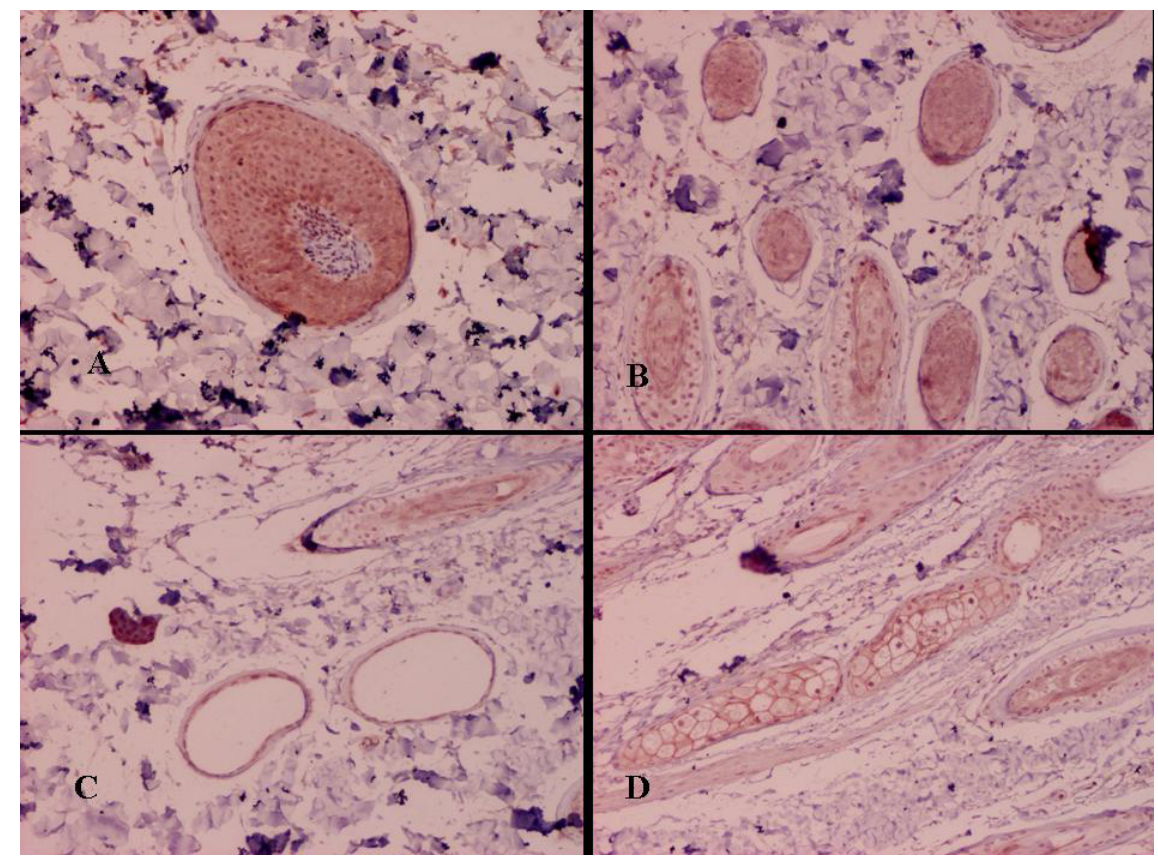

Figure 1. Expression of VEGF in epidermal appendages of adult female Liaoning cashmere goats (DAB immunoreaction, Bars on a-d $100 \mu \mathrm{m})$ VEGF was expressed in primary hair follicle (A) and secondary hair follicle (B); strong immunoreactivity for VEGF was seen between the hair matrix and the hair papilla, the follicular basement membrane zone (BMZ); VEGF was expressed in eccrine sweat glands (ductal and secretory portions) (C); VEGF was expressed in sebaceous glands (ductal and secretory portions) (D).

Average optical of VEGF-positive area in different months

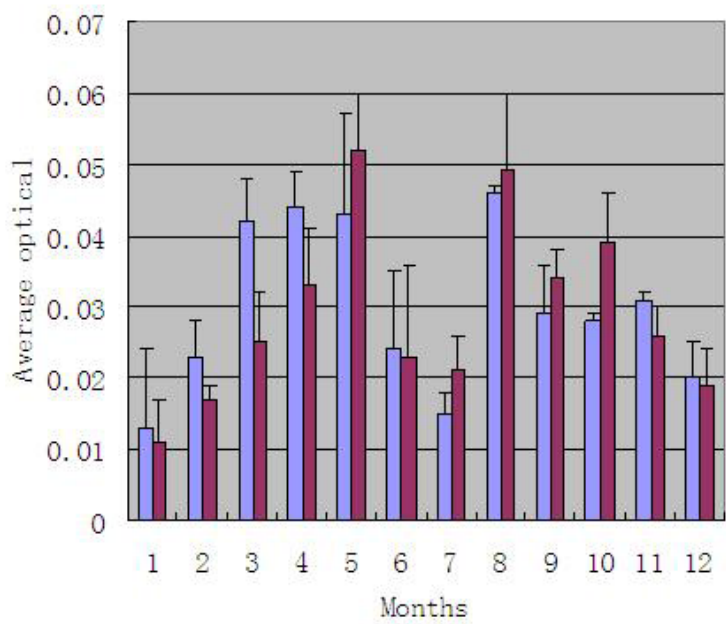

\author{
$\square$ primary hair follicle
}

$\square$ secondary hair follicle

Months

Figure 2. Average optical of VEGF-positive area in hair follicle of adult female Liaoning cashmere goats in different months. 


\section{Quantification of MVD by immunoreaction with CD34}

Hair follicle vascularization appears to be closely related to the processes involved in the hair cycle, and indeed, MVD changed with hair follicle development (Figure 3). Interestingly, the change of MVD in the dermis also showed a bimodal pattern (Figure 4); two peaks were observed in May and August, while levels were stable across the other months $(\mathrm{P} \geq 0.05)$. There was a small crest value in March, and the difference was significant when compared to adjacent months $(\mathrm{P} \leq 0.05)$.

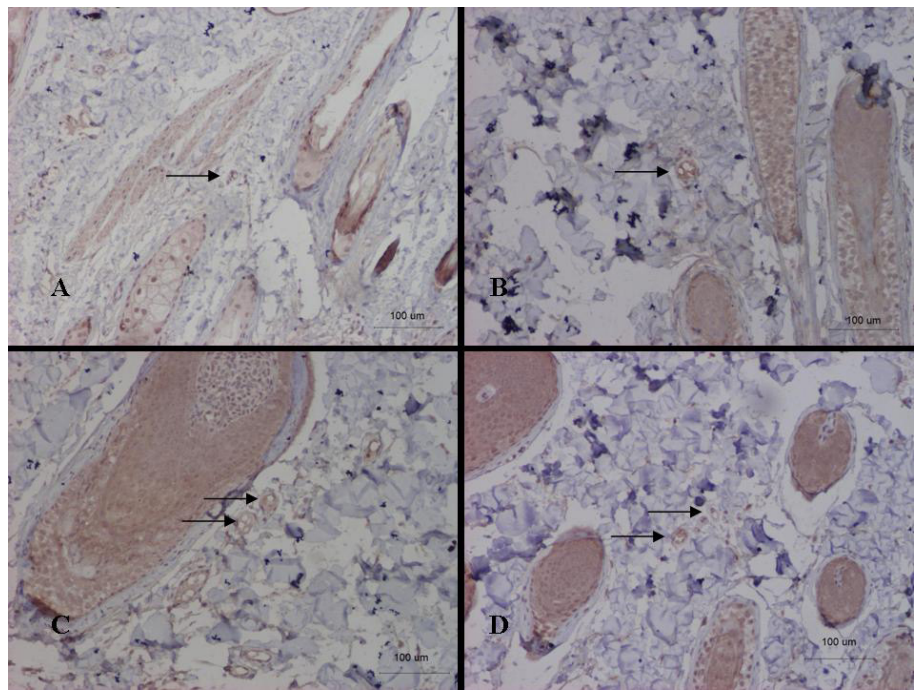

Figure 3. Microvessels distribution in skin of adult female Liaoning cashmere goats in different months (CD34, DAB immunoreaction, Bars on a-d $100 \mu \mathrm{m})$. Immunoreaction of MVD in skin of adult female Liaoning cashmere goats in January (A); immunoreaction of MVD in skin of adult female Liaoning cashmere goats in March (B); immunoreaction of MVD in skin of adult female Liaoning cashmere goats in May (C); immunoreaction of MVD in skin of adult female Liaoning cashmere goats in August (D).

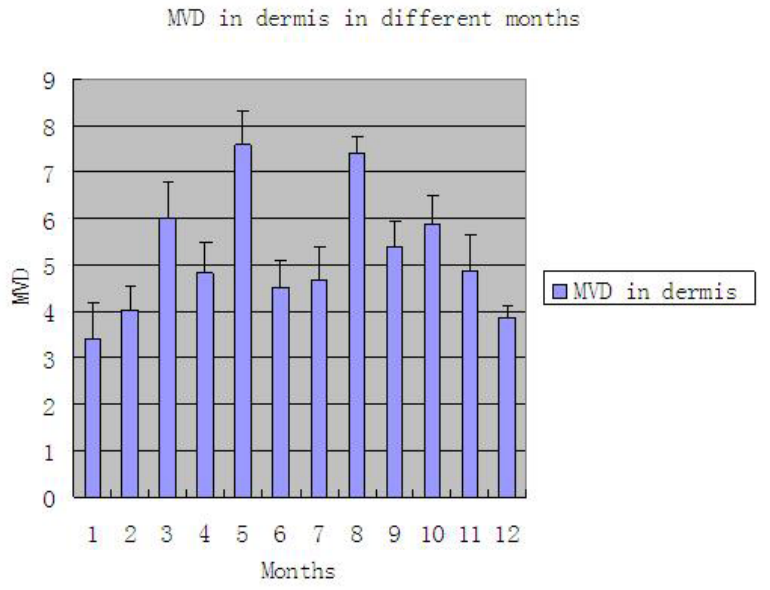

Figure 4. MVD in dermis of Liaoning cashmere goats' adult female in different months. 
Pearson correlation analysis showed that VEGF expression in primary hair follicles and MVD were moderately positively correlated $(\mathrm{r}=0.564, \mathrm{P}=0.000)$, while VEGF expression in secondary hair follicles and MVD were strongly positively correlated $(\mathrm{r}=0.703, \mathrm{P}=$ 0.000). Therefore, VEGF expression in secondary hair follicles had more of an effect on the MVD of the dermis than it did in primary hair follicles.

\section{DISCUSSION}

\section{Relationship between VEGF and MVD expression}

Hair follicle cycling is a developmental process that occurs over the total lifetime of a mammal. Whereas some researchers have suggested that all mature follicles undergo a growth cycle consisting of a growth phase (anagen), regression phase (catagen), rest phase (telogen), and shedding phase (exogen) (Stenn et al., 2001), others suggest that hair follicles instead undergo a growth cycle consisting of only the anagen, catagen, and telogen phases (Schneider et al., 2009). As manifest by its cyclical nature, the hair follicle is a regenerating system (Silver et al., 1977). The hair follicle first develops from the embryonic epidermis as an epithelial finger. This peg differentiates into three enclosed epithelial cylinders, forming the shaft (fiber), the ORS, and the IRS, and the shaft and IRS move outward together (Stenn et al., 2001). Both papilla fibroblasts and ORS keratinocytes contain angiogenic factors, such as the VEGF (Goldman et al., 1995; Kozlowska et al., 1998a; Lachgar et al., 1996b, 1998), a growth factor essential for vasculogenesis and angiogenesis (Carmeliet et al., 1996; Ferrara et al., 1996). The inferior hair follicle dramatically reforms itself over the cycle, whereas the upper, permanent follicle undergoes substantial remodeling (Lindner et al., 2000). This cyclic regeneration is believed to require many cellular signals that are also integral to other morphogenetic and regenerating systems.

Initially described by Judah Folkman in 1969, angiogenesis is an essential phase in uncontrolled cell proliferation, invasion, and metastasis, and is involved in the formation and remodeling of new vessels (Guster et al., 2010). The VEGF is a cytokine that plays a central role in angiogenesis, and is currently considered as one of the most important angiogenic factors. VEGFs are released by cells and induce formation of the capillary vessel network through receptor tyrosine kinase-mediated signaling. In normal skin, VEGF, which is expressed by epidermal and some dermal cells, is a likely regulator of vascular homeostasis (Viac et al., 1997). VEGF signals contain 3 specific receptors: VEGFR1, also known as Flt-1, VEGFR2 or Flk-1, and VEGFR3 (Press et al., 2007). MVD has been extensively evaluated as a measure of angiogenesis. Although MVD is not generally accepted as an established prognostic factor, it has been found to negatively affect survival in previous studies (Gaffney et al., 2003).

In the present study, angiogenesis was quantified using the antibody CD34 and expressed through the MVD. Angiogenesis was correlated to the hair follicle cycle and the VEGF, as the change of hair follicle development is concomitant with changes of VEGFs and MVD. The immunoreactivity analysis for VEGF showed that it was expressed in the hair bulb of primary and secondary hair follicles, the ORS, IRS, sebaceous glands (ductal and secretory portions), eccrine sweat glands (ductal and secretory portions), and epidermis. Man et al. (2009) also showed that VEGF was expressed in anagen hair follicles, including the inner or outer root sheath, fiber root sheath, dermal papillae, and progenitors of the hair shaft and 
pluripotential epidermal matrix cells. Furthermore, VEGF expression and the MVD increased during the anagen to catagen transition in the primary and secondary hair follicles, and the change in MVD and VEGF expression in the dermis showed bimodal patterns, in which two peaks were observed in May and August. Therefore, these results suggest that VEGF expression is similar in the primary and secondary hair follicles, and MVD is correlated to VEGF expression. Pearson correlation analysis showed that VEGF expression was moderately positively correlated $(\mathrm{r}=0.564, \mathrm{P}=0.000)$ with MVD in the primary hair follicles and strongly positively correlated with MVD in the secondary hair follicles $(\mathrm{r}=0.703, \mathrm{P}=0.000)$. We concluded that VEGF expression in secondary hair follicles had more important effects on MVD of the dermis than it did in primary hair follicles. VEGFs might contribute to hair growth by acting directly on papilla cells or by stimulating local vascularization. That is, cyclical hair growth might depend on the ability of the dermal papilla to synthesize and release soluble growth and differentiation factors toward pre-existing capillaries (Lachgar et al., 1996b). With the recycling of hair follicles, the number and shape of acroteric microvessels changes, mainly because VEGF expression in hair follicles is controlled by the hair follicle (Jiang et al., 2010).

VEGFs act as mitogens for endothelial cells that induce the formation of lumen vessels and increase vascular permeability and protein extravasation (Dvorak et al., 1995). It was previously demonstrated that estrogen increases VEGF concentration (Charnock-Jones et al., 1993), suggesting that VEGF expression should be higher in females than in males. However, this could not be confirmed in our experiment since we only used female Liaoning cashmere goats.

\section{ACKNOWLEDGMENTS}

This research was supported by the Cashmere Goat Breeding Center of Liaoning Province. This study was also supported by grants from the National Natural Science Foundation of China (NSFC; \#308871792, \#30800807, \#31072097), the Ministry of Science and Technology Support Program of China (\#2009BADA5B03), and the Special Postdoctoral Foundation of the Ministry of Education of China (\#2013T60332) and Special Funds for Scientific Research on Public Causes (\#201303119).

\section{REFERENCES}

Ballaun C, Weninger W, Uthman A, Weich H, et al. (1995). Human keratinocytes express the three major splice forms of vascular endothelial growth factor. J. Invest. Dermatol. 104: 7-10.

Borodach GN and Montagna W (1956) Fat in skin of the mouse during cycles of hair Growth1. J. Invest. Dermatol. 26 : 229-232.

Carmeliet P, Ferreira V, Breier G, Pollefeyt S, et al. (1996). Abnormal blood vessel development and lethality in embryos lacking a single $V E G F$ allele. Nature 380: 435-439.

Charnock-Jones DS, Sharkey AM, Rajput-Williams J, Burch D, et al. (1993). Identification and localization of alternately spliced mRNAs for vascular endothelial growth factor in human uterus and estrogen regulation in endometrial carcinoma cell lines. Biol. Reprod. 48: 1120-1128.

Chase R. (1965). Information system analysis of the organization of motor activity. Proced. Annu. Meet. Am. Psychopathol. Assoc. 53: 83-103.

Couchman JR (1986). Rat hair follicle dermal papillae have an extracellular matrix containing basement membrane components. J. Invest. Dermatol. 87: 762-767.

Custer SK, Neumann M, Lu H, Wright AC, et al. (2010). Transgenic mice expressing mutant forms VCP/p97 recapitulate the full spectrum of IBMPFD including degeneration in muscle, brain and bone. Hum. Mol. Genet. 19:1741-1755. 
Dvorak HF, Brown LF, Detmar M and Dvorak AM (1995). Vascular permeability factor/vascular endothelial growth factor, microvascular hyperpermeability, and angiogenesis. Am. J. Pathol. 146: 1029-1039.

Ellis RA and Moretti G (1959) Vascular patterns associated with catagen hair follicles in the human scalp *. Ann. N. Y. Acad. Sci. 83: 448-457.

Ferrara N, Carver-Moore K, Chen H, Dowd M, et al. (1996). Heterozygous embryonic lethality induced by targeted inactivation of the VEGF gene. Nature 380: 439-442.

Ferrara N, Gerber HP and LeCouter J (2003). The biology of VEGF and its receptors. Nat. Med. 9: 669-676.

Gaffney DK, Haslam D, Tsodikov A, Hammond E, et al. (2003). Epidermal growth factor receptor (EGFR) and vascular endothelial growth factor (VEGF) negatively affect overall survival in carcinoma of the cervix treated with radiotherapy. Int. J. Radiat. Oncol. Biol. Phys. 56: 922-928.

Goldman CK, Tsai JC, Soroceanu L and Gillespie GY (1995). Loss of vascular endothelial growth factor in human alopecia hair follicles. J. Invest. Dermatol. 104: 18S-20S.

Jiang HZ, Chen Y and Chang Q (2010). Effect of vascular endothelial growth factor (VEGF) on regulation of Angiogenesis around skin follicle of mammal. China Anim. Husbandry Vet. Med. 37: 47-49.

Kozlowska U, Blume-Peytavi U, Kodelja V, Sommer C, et al. (1998a). Expression of vascular endothelial growth factor (VEGF) in various compartments of the human hair follicle. Arch. Dermatol. Res. 290: 661-668.

Kozlowska U, Blume-Peytavi U, Kodelja V, Sommer C, et al. (1998b). Vascular endothelial growth factor expression induced by proinflammatory cytokines (interleukin 1 alpha, beta) in cells of the human pilosebaceous unit. Dermatology 196: 89-92.

Lachgar S, Charveron M, Gall Y, Plouet J, et al. (1996a). Vascular endothelial cells: targets for studying the activity of hair follicle cell-produced VEGF. Cell Biol. Toxicol. 12: 331-334.

Lachgar S, Moukadiri H, Jonca F, Charveron M, et al. (1996b). Vascular endothelial growth factor is an autocrine growth factor for hair dermal papilla cells. J. Invest. Dermatol. 106: 17-23.

Lachgar S, Charveron M, Gall Y and Bonafe JL (1998). Minoxidil upregulates the expression of vascular endothelial growth factor in human hair dermal papilla cells. Br. J. Dermatol. 138: 407-411.

Lindner G, Menrad A, Gharadi E, Merlino G, et al. (2000). Involvement of hepatocyte growth factor/scatter factor and receptor signalling in hair follicle morphogenesis and cycling. FASEB J. 14: 319-332.

Majewski S, Tokarski G and Moskalewski S (1985). Relationship between the hair growth cycle and the intensity of lymphocyte-induced angiogenesis in mouse skin. Arch. Dermatol. Res. 277:77-78.

Man XY, Yang XH, Cai SQ, Bu ZY, et al. (2009). Expression and localization of vascular endothelial growth factor and vascular endothelial growth factor receptor-2 in human epidermal appendages: a comparison study by immunofluorescence. Clin. Exp. Dermatol. 34: 396-401.

Parakkal PF (1970). Morphogenesis of the hair follicle during catagen. Z. Zellforsch. Mikrosk Anat. 107:174-186.

Press MF and Lenz HJ (2007). EGFR, HER2 and VEGF pathways: validated targets for cancer treatment. Drugs 67: 2045-2075.

Reynolds AJ and Jahoda CA (1996). Hair matrix germinative epidermal cells confer follicle-inducing capabilities on dermal sheath and high passage papilla cells. Development 122: 3085-3094.

Roy H, Bhardwaj S and Yla-Herttuala S (2006). Biology of vascular endothelial growth factors. FEBS Lett. 580: 28792887.

Schneider MR, Schmidt-Ullrich R and Paus R (2009). The hair follicle as a dynamic miniorgan. Curr. Biol. 19: 132-142.

Silver AF and Chase HB (1977). The incorporation of tritiated uridine in hair germ and dermal papilla during dormancy (telogen) and activation (early anagen). J. Invest. Dermatol. 68: 201-205.

Stenn KS and Paus R (2001). Controls of hair follicle cycling. Physiol. Rev. 81: 450-481.

Tammela T, Enholm B, Alitalo K and Paavonen K (2005). The biology of vascular endothelial growth factors. Cardiovasc. Res. 65: 550-563.

Viac J, Palacio S, Schmitt D and Claudy A (1997). Expression of vascular endothelial growth factor in normal epidermis, epithelial tumors and cultured keratinocytes. Arch. Dermatol. Res. 289: 158-163.

Weninger W, Uthman A, Pammer J, Pichler A, et al. (1996). Vascular endothelial growth factor production in normal epidermis and in benign and malignant epithelial skin tumors. Lab. Invest. 75: 647-657. 